\title{
Vertex representations of quantum affine algebras
}

\author{
(Yang-Baxter equation/Hopf algebras/q-analogues/Chevalley basis/basic representations)
}

\author{
IgOR B. Frenkel and Naihuan Jing
}

Department of Mathematics, Yale University, New Haven, CT 06520

Communicated by G. D. Mostow, September 6, 1988

\begin{abstract}
We construct vertex representations of quantum affine algebras of ADE type, which were first introduced in greater generality by Drinfeld and Jimbo. The limiting special case of our construction is the untwisted vertex representation of affine Lie algebras of Frenkel-Kac and Segal. Our representation is given by means of a new type of vertex operator corresponding to the simple roots and satisfying the defining relations. In the case of the quantum affine algebra of type $A$, we introduce vertex operators corresponding to all the roots and determine their commutation relations. This provides an analogue of a Chevalley basis of the affine Lie algebra $\widehat{\mathfrak{S I}}(n)$ in the basic representation.
\end{abstract}

\section{Section 1. Introduction}

The quantum Yang-Baxter equation arises in a number of problems of quantum field theory and statistical mechanics. Different aspects of this equation, related problems, and a mathematical foundation can be found in a recent review (1), which also contains references to the early physics literature. Additional interest in the quantum Yang-Baxter equation has been stirred by the independent discovery of Drinfeld (2) and of Jimbo (3) of a fundamental algebraic structure underlying its solutions (see also ref. 4 and references therein). Drinfeld and Jimbo defined a quantum or quantized Kac-Moody algebra which may be thought of as a $q$-analogue or $q$-deformation of the universal enveloping algebra of a Kac-Moody algebra, introduced in refs. 5-7. They also showed that these algebras are Hopf algebras and thus allow the tensor product structure on their representations. Dominant highest weight representations for Kac-Moody algebras were first constructed by Kac (8), who also generalized Weyl's character formula. Lusztig (9) has shown that these representations can be deformed consistently with a $q$-deformation of Kac-Moody algebras into the Drinfeld-Jimbo algebras.

In the first,works on Kac-Moody algebras $(5,6)$, it was realized that the algebras associated with extended Cartan matrices admit a simple realization as simple Lie algebras over Laurent polynomials often called (centerless) affine Lie algebras or loop algebras. In ref. 10 Drinfeld found a new realization of the Drinfeld-Jimbo algebras associated with extended Cartan matrices, which we will call quantum affine algebras. A realization of the untwisted basic representations of affine Lie algebras by means of vertex operators in Fock spaces was reported in refs. 11 and 12, following the first twisted construction of ref. 13. The present work is a generalization of the untwisted realization to the case of quantum affine algebras.

In Section 2 we recall the definition of the Drinfeld-Jimbo algebras corresponding to the extended Cartan matrices of types $\hat{A}_{l}, \hat{D}_{l}$ and $\hat{E}_{l}$. Then we give Drinfeld's realization of

The publication costs of this article were defrayed in part by page charge payment. This article must therefore be hereby marked "advertisement" in accordance with 18 U.S.C. $\$ 1734$ solely to indicate this fact. these algebras. In Section 3 we describe the vertex representation of the quantum affine algebras of ADE types. First we construct a Fock space which, in agreement with Lusztig's general result, has the same graded dimension as its affine Lie algebra analogue. Then we introduce a type of vertex operator that corresponds to the simple roots and we show that these operators satisfy the correct relations of Drinfeld's realization. It is instructive to note that Drinfeld's realization uses the generators corresponding to the affinization of the simple roots of finite type, while the realization of affine $\mathrm{Lie}$ algebras provides generators corresponding to all the affine roots, which appropriately normalized provide a Chevalley basis. In Section 4 we construct a Chevalley basis for the quantum affine algebra of type $\hat{A}_{l}$ in the basic representations by means of a further generalization of vertex operators.

One cannot avoid a remarkable parallel between the development of the theories of affine Lie algebras and quantum affine algebras, thus implementing another direction in close relation to but beyond the affine Lie algebras (14). Exploring further this parallel we recall that the vertex representation of affine Lie algebras leads to the simplest example of vertex operator algebras $(15,16)$ and, consequently, of conformal field theories (17). The generalization of these theories to the quantum case is an extensive program which can lead to important discoveries and relations. In particular, the quantum analogue of the vertex operator algebra and conformal field theory associated to the discrete series representations of the Virasaro algebra $(18,19)$ could shed more light on the relation of these representations to two-dimensional statistical models and the quantum Yang-Baxter equation.

\section{Section 2. Quantum Affine Algebras}

We recall the definition of quantum affine algebras in terms of generators and relations $(2,3)$. In this paper, we consider only the simply laced case-i.e., that in which all the roots have the same length.

Let $\Gamma$ be a Dynkin diagram of type $A_{l}(l \geq 1), D_{l}(l \geq 4)$, or $E_{l}(l=6,7$, or 8$)$, and let $\Gamma=\Gamma \cup\{0\}$ be the extended Dynkin diagram corresponding to $\Gamma$. We denote by $A=\left(A_{i j}\right)_{i, j=1}^{l}$ and $\hat{A}=\left(A_{i j}\right)_{i, j=0}^{l}$ the Cartan matrices associated to $\Gamma$ and $\hat{\Gamma}$, respectively. In the simply laced case $A_{i j}=0,-1$, or \pm 2 . We denote by $\mathfrak{g}$ and $\hat{\mathrm{g}}$ the Kac-Moody Lie algebras over $\mathbb{C}$ constructed via the Cartan matrices $A$ and $\hat{A}$, respectively, and by $\Delta$ and $\hat{\Delta}$ the corresponding root systems. Let $\alpha_{1}, \ldots, \alpha_{l}$ be a basis of $\Delta$; a basis of $\hat{\Delta}$ contains an additional element $\alpha_{0}$. We introduce a symmetric bilinear form $\langle$,$\rangle in the vector$ space with basis $\alpha_{i}, i=0,1, \ldots, l$, such that $\left\langle\alpha_{i}, \alpha_{j}\right\rangle=A_{i j}, i$, $j=0,1, \ldots, l$.

The quantum affine algebra $U(\hat{\mathrm{g}})_{q}$ corresponding to $\hat{\Gamma}$ is an analogue of the universal enveloping algebra of $\hat{\mathrm{g}}$. It is an associative algebra with unit 1 and generators

$$
\left\{x_{i}^{+}, x_{i}^{-}, k_{i}, k_{i}^{-1} \quad i=0,1, \ldots, l\right\}
$$

satisfying the relation 


$$
\begin{array}{r}
k_{i} k_{i}^{-1}=k_{i}^{-1} k_{i}=1, \quad\left[k_{i}, k_{j}\right]=0 \\
k_{i} x_{j}^{ \pm} k_{i}^{-1}=q^{ \pm A_{i j}} x_{j}^{ \pm} \\
{\left[x_{i}^{+}, x_{j}^{-}\right]=\delta_{i j}\left(k_{i}-k_{i}^{-1}\right) /\left(q-q^{-1}\right)} \\
{\left[x_{i}^{ \pm}, x_{j}^{ \pm}\right]=0 \text { for } A_{i j}=0} \\
x_{i}^{ \pm} x_{i}^{ \pm} x_{j}^{ \pm}-\left(q+q^{-1}\right) x_{i}^{ \pm} x_{j}^{ \pm} x_{i}^{ \pm}+x_{j}^{ \pm} x_{i}^{ \pm} x_{i}^{ \pm} \\
=0 \text { for } A_{i j}=-1,
\end{array}
$$

where $i, j=0,1, \ldots, l$ and $q$ is a nonzero complex number, $q \neq \pm 1$. We will also use its logarithm $t / 2$ :

$$
q=e^{t / 2} .
$$

In the case when $\hat{\Gamma}$ is of type $\hat{A}_{1}$, the defining relations $\mathbf{2 . 6}$ are replaced by a fourth-order relation for $A_{i j}=-2$ (see refs. 2 and 3 for the explicit formula).

Let $\alpha_{\max }=n_{1} \alpha_{1}+\ldots+n_{l} \alpha_{l}$ be a decomposition of the maximal root with respect to the basis of simple roots. Then $c=\alpha_{0}+n_{1} \alpha_{1}+\ldots+n_{l} \alpha_{l}$ is a central element of the affine Lie algebra $\hat{g}$. Similarly,

$$
\gamma=k_{0} k_{1}^{n_{1}} \ldots k_{l}^{n_{l}}
$$

is a central element in $U(\hat{\mathrm{g}})_{q}$.

To define a Hopf algebra structure on $U(\hat{\mathrm{g}})_{q}$, one also needs to introduce "square roots" $k_{i}^{1 / 2}, k_{i}^{-1 / 2}, i=0,1, \ldots, l$, with obvious replacements of relations (2.2 and 2.3) [see Jimbo (3)]. We will always adjoin $\gamma^{1 / 2}, \gamma^{-1 / 2}$ to $U(\hat{\mathrm{g}})_{q}$. Drinfeld defines a quantum affine algebra by introducing "logarithmic" generators $\alpha_{i}$ such that $k_{i}=e^{t \alpha_{i} / 2}, i=0,1, \ldots, l$; hence $\gamma=e^{t c / 2}$. In this form the quantum affine algebra $U(\hat{\mathrm{g}})_{q}$ for $t$ $\rightarrow 0(q \rightarrow 1)$ degenerates into the universal enveloping algebra of the affine Lie algebra.

The subalgebra $U(\mathrm{~g})_{q}$ of $U(\hat{\mathrm{g}})_{q}$ generated by $x_{i}^{+}, x_{i}, k_{i}, k_{i}^{-1}$, for $i=1, \ldots, l$ is the quantum analogue of the universal enveloping algebra $U(\mathrm{~g})$ of the finite-dimensional simple Lie algebra $\mathrm{g}$ corresponding to $\Gamma$. For $t \rightarrow 0(q \rightarrow 1) U(\mathfrak{g})_{q}$ degenerates into $U(\mathrm{~g})$.

Remark: There is a minor discrepancy between the Drinfeld and Jimbo form for relation 2.4. Namely, Drinfeld uses $t$ instead of $q-q^{-1}=2 \sinh t / 2$ in the denominator, which implies corresponding changes in other relations (see, e.g., the denominator of relation $\mathbf{2 . 2 2}$ below). However, the vertex representation will yield Jimbo's form, which we will use in this paper.

Drinfeld discovered in ref. 10 a remarkable realization of quantum affine algebras which is to a great extent parallel to the realization of affine Lie algebras in the form $\hat{g} \simeq g \otimes \mathbb{C}[$, $\left.t^{-1}\right] \otimes \mathbb{C} c$ with the central element $c$ and the standard Lie bracket (see, e.g., ref. 11). In fact Drinfeld's realization can be thought of as an affinization of the quantum finite-dimensional algebra $U(\mathrm{~g})_{q}$ with the generators 2.1, $i=1, \ldots, l$, and relations 2.2-2.6 written with the help of a universal function $g_{i j}(z), i, j=1, \ldots, l$ defined as follows. Let $\alpha, \beta \in$ $\Delta$. We set

$$
g_{\alpha \beta}(z)=\sum_{n \in \mathbb{Z}_{+}} \mathfrak{c}_{\alpha \beta n} z^{n}
$$

a formal power series in $z$, where the coefficients $c_{\alpha \beta n}$ are determined from the Taylor series expansion in the variable $\zeta$ at $0 \in \mathbb{C}$ of the function

$$
f_{\alpha \beta}(\zeta)=\left(q^{\langle\alpha, \beta\rangle} \zeta-1\right) /\left(\zeta-q^{\langle\alpha, \beta\rangle}\right)=\sum_{n \in \mathbb{Z}_{+}} c_{\alpha \beta n} \zeta^{n} .
$$

We note that while $f_{\alpha \beta}\left(\zeta^{-1}\right)=f_{\alpha \beta}(\zeta)^{-1}$ this is not true for $g_{\alpha \beta}(z)$. We set $g_{i j}(z)=g_{\alpha_{i} \alpha_{j}}(z), i, j=1, \ldots, l$. We also define a formal power series

$$
\delta(z)=\sum_{n \in \mathbb{Z}} z^{n} .
$$

Now we can formulate a slight hidifification of Drinfeld's realization for the simply laced case.

DRINFELD's THEOREM: The quantum affine algebra $\mathrm{U}(\hat{\mathrm{g}})_{\mathrm{a}}$ is isomorphic to the associative algebra with unit 1 and the generators

$\left\{\mathrm{x}_{\mathrm{ik}}^{ \pm}, \varphi_{\mathrm{im}}, \psi_{\mathrm{in}}, \gamma^{1 / 2}, \gamma^{-1 / 2} \mid \mathrm{i}=1, \ldots, \mathrm{l} ;\right.$

$$
\left.\mathrm{k} \in \mathbb{Z}, \mathrm{m} \in-\mathbb{Z}_{+}, \mathrm{n} \in \mathbb{Z}_{+}\right\}
$$

satisfying the relations below written in terms of the following generating functions in a formal variable $\mathrm{z}$ :

$$
\begin{aligned}
& x_{i}^{ \pm}(z)=\sum_{k \in \mathbb{Z}} x_{i k}^{ \pm} z^{-k} \\
& \varphi_{i}(z)=\sum_{m \in-Z_{+}} \varphi_{i m} z^{-m} \\
& \psi_{i}(z)=\sum_{n \in Z_{+}} \psi_{i n} z^{-n} .
\end{aligned}
$$

Written in terms of formal variables, the relations are

$$
\begin{aligned}
& \gamma^{1 / 2} \gamma^{-1 / 2}=\gamma^{-1 / 2} \gamma^{1 / 2}=1, \quad \gamma^{ \pm 1 / 2} \text { are central } \\
& \varphi_{\mathrm{io}} \psi_{\mathrm{io}}=\psi_{\mathrm{io}} \varphi_{\mathrm{io}}=1 \\
& {\left[\varphi_{\mathrm{i}}(\mathrm{z}), \varphi_{\mathrm{j}}(\mathrm{w})\right]=0, \quad\left[\psi_{\mathrm{i}}(\mathrm{z}), \psi_{\mathrm{j}}(\mathrm{w})\right]=0} \\
& \varphi_{\mathrm{i}}(\mathrm{z}) \psi_{\mathrm{j}}(\mathrm{w}) \varphi_{\mathrm{i}}(\mathrm{z})^{-1} \psi_{\mathrm{j}}(\mathrm{w})^{-1}=\mathrm{g}_{\mathrm{ij}}\left(\mathrm{zw}^{-1} \gamma^{-1}\right) / \mathrm{g}_{\mathrm{ij}}\left(\mathrm{zw}^{-1} \gamma\right) \\
& \varphi_{\mathrm{i}}(\mathrm{z}) \mathrm{x}_{\mathrm{j}}^{ \pm}(\mathrm{w}) \varphi_{\mathrm{i}}(\mathrm{z})^{-1}=\mathrm{g}_{\mathrm{ij}}\left(\mathrm{zw}^{-1} \gamma^{\mp 1 / 2}\right)^{ \pm 1} \mathrm{x}_{\mathrm{j}}^{ \pm}(\mathrm{w}) \\
& \psi_{\mathrm{i}}(\mathrm{z}) \mathrm{x}_{\mathrm{j}}^{ \pm}(\mathrm{w}) \psi_{\mathrm{i}}(\mathrm{z})^{-1}=\mathrm{g}_{\mathrm{ij}}\left(\mathrm{z}^{-1} \mathrm{w} \gamma^{\mp 1 / 2}\right)^{\mp 1} \mathrm{x}_{\mathrm{j}}^{ \pm}(\mathrm{w}) \\
& {\left[\mathrm{x}_{\mathrm{i}}^{+}(\mathrm{z}), \mathrm{x}_{\mathrm{j}}^{-}(\mathrm{w})\right]=\delta_{\mathrm{ij}}\left\{\delta\left(\mathrm{zw}^{-1} \gamma^{-1}\right) \psi_{\mathrm{i}}\left(\mathrm{w} \gamma^{1 / 2}\right)\right.} \\
& \left.-\delta\left(\mathrm{zw}^{-1} \gamma\right) \varphi_{\mathrm{i}}\left(\mathrm{z} \gamma^{1 / 2}\right)\right\} /\left(\mathrm{q}-\mathrm{q}^{-1}\right) \\
& \left(z-q^{ \pm A_{i j}} w\right) x_{i}^{ \pm}(z) x_{j}^{ \pm}(w)=\left(q^{ \pm A_{i j}} z-w\right) x_{j}^{ \pm}(w) x_{i}^{ \pm}(z) \\
& {\left[\mathrm{x}_{\mathrm{i}}^{ \pm}(\mathrm{z}), \mathrm{x}_{\mathrm{j}}^{ \pm}(\mathrm{w})\right]=0 \text { for } \mathrm{A}_{\mathrm{ij}}=0} \\
& \left\{x_{i}^{ \pm}\left(z_{1}\right) x_{i}^{ \pm}\left(z_{2}\right) x_{j}^{ \pm}(w)-\left(q+q^{-1}\right) x_{i}^{ \pm}\left(z_{1}\right) x_{j}^{ \pm}(w) x_{i}^{ \pm}\left(z_{2}\right)\right. \\
& \left.+\mathbf{x}_{\mathbf{j}}^{ \pm}(\mathrm{w}) \mathrm{x}_{\mathbf{i}}^{ \pm}\left(\mathrm{z}_{1}\right) \mathrm{x}_{\mathbf{i}}^{ \pm}\left(\mathrm{z}_{2}\right)\right\}+\left\{\mathrm{z}_{1} \leftrightarrow \mathrm{z}_{2}\right\}=0 \\
& \text { for } \mathrm{A}_{\mathrm{ij}}=-1 .
\end{aligned}
$$

The latter relations (2.23-2.25) should be replaced by a fourth-order relation (10) for $\hat{\Gamma}=\hat{\mathrm{A}}_{1}$.

An explicit isomorphism between the two presentations of $U(\hat{\mathrm{g}})_{q}$ is given by the following.

$$
x_{i}^{ \pm} \rightarrow x_{i o}^{ \pm}, \quad k_{i} \rightarrow \psi_{i o}, \quad k_{i}^{-1} \rightarrow \varphi_{i o}
$$

for $i=1, \ldots, l, k_{0}, k_{0}^{-1}$ are determined from relation 2.8 and some appropriate formulas for $x_{0}^{+}, x_{0}^{-}$, which can be found in ref. 10. We will return to explicit expressions of $x_{0}^{ \pm}$in relation to the Chevalley basis in Section 4.

Again for the Hopf algebra structure on $U(\hat{\mathrm{g}})_{q}$ one needs to introduce "square roots" $\varphi_{i o}^{1 / 2}, \psi_{i o}^{1 / 2}, i=1, \ldots, l$. One can also introduce "logarithmic" generators $\alpha_{i o}$, where $\psi_{i o}=$ $e^{t \alpha_{i o} / 2}, \varphi_{i o}=e^{-t \alpha_{i} / 2}, i=1, \ldots, l$, and $c$, where $\gamma=e^{t c / 2}$ as in the definition of $U(\hat{\mathrm{g}})_{q}$. It is also useful to define elements $\alpha_{i n}, i=1, \ldots, l, n \in \mathbb{Z}, n \neq 0$, from the relations 


$$
\begin{aligned}
& \varphi_{i}(z)=\varphi_{i o} \exp \left\{-t\left(\sum_{m \in-\mathbf{Z}_{+}} \alpha_{i m} z^{-m}\right)\right\} \\
& \psi_{i}(z)=\psi_{i o} \exp \left\{t\left(\sum_{n \in Z_{+}} \alpha_{i n} z^{-n}\right)\right\}
\end{aligned}
$$

In fact, equating the homogeneous components in the formal power series in $z$, one gets an inductive definition of $\alpha_{i n}$ in terms of $\varphi_{i n}, \psi_{i n}$. One can then deduce the relations for $\alpha_{i n}^{ \pm}$ from the corresponding relations for $\varphi_{i}(z), \psi_{i}(z)$. Taking the logarithm of the right-hand side of relation 2.19 and passing to the component form, one obtains for $m, n \neq 0$

$\left[\alpha_{i m}, \alpha_{j n}\right]=\delta_{m,-n} \frac{1}{m t^{2}}\left(q^{m A_{i j}}-q^{-m A_{i j}}\right)\left(\gamma^{m}-\gamma^{-m}\right)$.

Similarly, for $m \neq 0$ one gets

$$
\left[\alpha_{i m}, x_{j}^{ \pm}(z)\right]= \pm \frac{z^{m}}{m t}\left(q^{m A_{i j}}-q^{-m A_{i j}}\right) \gamma^{\mp|m| / 2} x_{j}^{ \pm}(z) .
$$

The two latter commutators (2.29 and 2.30), though not as elegant as the corresponding relations (2.19-2.21), will be crucial in our construction of the vertex representation.

\section{Section 3. Vertex Representations}

We recall that the basic representations of the simply laced affine Lie algebras can be defined as the dominant highest weight representations which admit the smallest positive value of the central element $c$, usually normalized to be 1 . An abstract construction of dominant highest weight representations of the Drinfeld-Jimbo algebras has been obtained in ref. 9. In this section we generalize the untwisted vertex construction of basic representations of the affine Lie algebras $(11,12)$ to the case of quantum affine algebras. We will assume that $q$ is not a root of unit.

Let $U(\hat{\mathfrak{h}})_{q}$ be the subalgebra of $U(\hat{\mathfrak{g}})_{q}$ generated by $\varphi_{i m}$, $\psi_{\text {in }}, \gamma, \gamma^{-1}, i=1, \ldots, l, m \in-\mathbb{Z}_{+}, n \in \mathbb{Z}_{+}$. We call $U(\hat{\mathfrak{h}})_{q}$ the quantum Heisenberg algebra. We denote by $U\left(\hat{\mathfrak{h}}^{+}\right)_{q}($ resp $\left.U\left(\mathfrak{h}^{-}\right)_{q}\right)$ the commutative subalgebra of $U(\hat{\mathfrak{h}})_{q}$ generated by $\psi_{i n}, \gamma, \gamma^{-1}, n \in \mathbb{Z}_{+},\left(\operatorname{resp} \varphi_{i m}, m \in-\mathbb{Z}_{+}\right), i=1, \ldots, l$. Since for every $n \in \mathbb{Z}_{+}, \alpha_{i n}$ is a polynomial in $\psi_{i n^{\prime}}, n^{\prime} \in \mathbb{Z}_{+}$and vice versa, we have the identification

$$
U\left(\mathfrak{h}^{-}\right)_{q} \simeq S\left(\mathfrak{h}^{-}\right)
$$

Let $\mathbb{C} 1_{s}$ be a one-dimensional representation of $U\left(\hat{\mathfrak{b}}^{+}\right)_{q}$ such that $\gamma$ acts as multiplication by $q$ (i.e., $c$ is 1 ), $\gamma^{-1}$ acts as $q^{-1}$, and the other generators act trivially. By the Poincaré-Birkhoff-Witt theorem for $U(\hat{\mathfrak{h}})_{q}$ and relation 3.1, one has

$$
U(\hat{\mathfrak{h}})_{q} \underset{U\left(\hat{\mathfrak{G}}^{+}\right)_{q}}{\otimes} \mathbb{C} 1_{s} \simeq S\left(\mathfrak{h}^{-}\right)
$$

We denote by $\alpha_{i}(k), \Phi_{i}(m), \Psi_{i}(n)$ the operators in $S\left(\mathfrak{h}^{-}\right)$representing the generators $\alpha_{i k}, \varphi_{i m}, \psi_{i n}$, respectively. One can easily obtain their explicit forms; namely, $\alpha_{i}(k)$ acts by multiplication by $\alpha_{i k}$ for $k<0$ and, by a derivation for $k>0$ satisfying the Heisenberg relations,

$\left[\alpha_{i}(m), \alpha_{j}(n)\right]=\delta_{m,-n} \frac{1}{m t^{2}}\left(q^{m A_{i j}}-q^{-m A_{i j}}\right)\left(q^{m}-q^{-m}\right),[3.3]$

and the operators $\Phi_{i}(m), \Psi_{i}(n)$ are determined from the relations

$$
\begin{aligned}
& \Phi_{i}(z)=\sum_{m \in-\mathbb{Z}_{+}} \Phi_{i}(m) z^{-m}= \\
& \exp \left\{-t\left(\frac{\alpha_{i}(0)}{2}+\sum_{m \in-\mathbb{Z}_{+}} \alpha_{i}(m) z^{-m}\right)\right\}, \\
& \Psi_{i}(z)=\sum_{n \in \mathbb{Z}_{+}} \Psi_{i}(n) z^{-n}= \\
& \exp \left\{t\left(\frac{\alpha_{i}(0)}{2}+\sum_{n \in \mathbb{Z}_{+}} \alpha_{i}(n) z^{-n}\right)\right\},
\end{aligned}
$$

where the action of $\alpha_{i}(0)$ is defined below (see relation 3.9). The abuse of notation in relations 3.4 and 3.5 should not lead to any uncertainty since we always denote formal variables by $z, w$ and integers by $k, m, n$.

Let $Q$ be the root lattice of the simple simply laced finitedimensional Lie algebra corresponding to the Dynkin diagram $\Gamma$. We identify the weight lattice $P$ with the dual of $Q$. Then $P=\cup_{r}\left(Q+\omega_{r}\right)$ is the coset decomposition with respect to $Q$ where $\omega_{r}$ is a unique highest weight in a given coset such that $\left\langle\alpha_{\max }, \omega_{r}\right\rangle \leq 1$. Let $\hat{Q}$ be the central extension of $Q$ by the group $\langle \pm 1\rangle$

$$
1 \rightarrow\langle \pm 1\rangle \rightarrow \hat{Q} \stackrel{-}{\longrightarrow} Q \rightarrow 1
$$

with the commutator

$$
a b a^{-1} b^{-1}=(-1)^{\langle\bar{a}, \bar{b}\rangle}, \quad a, b \in \hat{Q} .
$$

We fix $a_{i} \in \hat{Q}, i=1, \ldots, l$, such that $\overline{a_{i}}=\alpha_{i}$. One could fix a section $Q \rightarrow \hat{Q}$ and define the corresponding 2-cocycle $\varepsilon: Q$ $\times Q \rightarrow\langle \pm 1\rangle$, but this is not necessary for our exposition.

Let $\mathbb{C 1} 1_{Q}$ be a natural one-dimensional representation of the group $\langle \pm 1\rangle$. We denote by $\mathbb{C}\{Q\}$ the induced $\hat{Q}$-module

$$
\mathbb{C}\{Q\}=\mathbb{C}[\hat{Q}] \underset{\mathbb{C}[\langle \pm 1\rangle]}{\otimes} \mathbb{C} 1_{Q},
$$

where $\mathbb{C}[G]$ denotes the group algebra of a group $G$. For fixed $r$, we will also view $\mathbb{C}\{Q\}$ as the representation of $U(\hat{\mathfrak{h}})_{q}$ trivial on the subalgebras $U\left(\hat{\mathfrak{h}}^{+}\right)_{q}$ and $U\left(\mathfrak{h}^{-}\right)_{q}$ and such that

$$
\alpha_{i}(0) a=\left\langle\alpha_{i}, \bar{a}+\omega_{r}\right\rangle a, \text { for } a \in \hat{Q}, i=1, \ldots, l .
$$

We will denote this representation by $\mathbb{C}\{Q\}_{r}$. We will also view $S\left(\mathfrak{h}^{-}\right)$as a trivial representation of $\hat{Q}$. For the highest weight $\omega_{r}$ (and $c=1$ ) we define the space of the basic representation of the quantum affine algebra $U(\hat{\mathrm{g}})_{q}$ as in the Lie algebra case:

$$
V_{r}=S\left(\mathfrak{h}^{-}\right) \otimes \mathbb{C}\{Q\}_{r} .
$$

By the above definition $V_{r}$ is a representation of $U(\hat{\mathfrak{h}})_{q}$ and $\hat{Q}$. We will extend it to the representation of the quantum affine algebra $U(\hat{\mathrm{g}})_{q}$ by means of new vertex operators. The explicit form of these vertex operators up to a factor $z^{ \pm \alpha_{i}(0)+1}$ can be determined from the commutation relation 2.30. For $i=1, \ldots, l$, one has

$$
\begin{aligned}
X_{i}^{ \pm}(z)= & \exp \left\{ \pm t \sum_{n \geq 1} \frac{q^{\mp n / 2}}{q^{n}-q^{-n}} \alpha_{i}(-n) z^{n}\right\} \\
& \times \exp \left\{\mp t \sum_{n \geq 1} \frac{q^{\mp n / 2}}{q_{n}-q^{-n}} \alpha_{i}(n) z^{-n}\right\} a_{i}^{ \pm 1} z^{ \pm \alpha_{i}(0)+1}
\end{aligned}
$$

$$
X_{i}^{ \pm}(z)=\sum_{n \in \mathbb{Z}} X_{i}^{ \pm}(n) z^{-n}
$$


While the vertex operators $X_{i}^{+}(z)$ map $V_{r}$ into the formal power series $V_{r}\{z\}$, its components $X_{i}^{ \pm}(n)$ are well-defined operators on $V_{r}$.

Remark: In the limit when $q \rightarrow 1(t \rightarrow 0)$ the vertex operators 3.11 coincide with the vertex operators providing the untwisted representation of affine Lie algebras $(11,12)$.

We will show that the operators $X_{i}^{ \pm}(n)$ provide a representation of the genrators $x_{i n}^{ \pm}$of the quantum affine algebra $U(\hat{g})_{q}$. Definition 3.11 implies relation 2.30 and therefore relations 2.20 and $\mathbf{2 . 2 1}$ with $\gamma=q$. In order to show the rest of the relations, we apply the standard technique of operator calculus in the formal variable approach or in the equivalent contour integral approach (both are explained in detail in ref. 16). As in the Lie algebra case, it is useful to define the normal ordering of an operator expressed in terms of $\alpha_{i}(n), a_{i}$. The only nontrivial orderings are

$$
\begin{aligned}
: \alpha_{i}(n) \alpha_{j}(-n): & =\alpha_{j}(-n) \alpha_{i}(n), \quad n \geq 0 \\
: \alpha_{i}(0) a_{j}: & =: a_{j} \alpha_{i}(0):=\frac{1}{2}\left(\alpha_{i}(0) a_{j}+a_{j} \alpha_{i}(0)\right)
\end{aligned}
$$

Then for example we obtain relation 2.22 using the following identities:

$$
\begin{aligned}
& X_{i}^{\mp}(z) X_{j}^{\ddagger}(w)=(1-w / z)(z / w)^{1 / 2}: X_{i}^{ \pm}(z) X_{j}^{\mp}(w):, \\
& \text { for } A_{i j}=-1 \\
& X_{i}^{\mp}(z) X_{i}^{ \pm}(w)=(1-w q / z)^{-1}(1-w / z q)^{-1}(w / z) \\
&: X_{i}^{\ddagger}(z) X_{j}^{\mp}(w): \\
&: X_{i}^{+}\left(z q^{-1}\right) X_{i}^{-}(z):=\Phi_{i}\left(z q^{-1 / 2}\right) \\
&: X_{i}^{+}(z q) X_{i}^{-}(z):=\Psi_{i}\left(z q^{1 / 2}\right),
\end{aligned}
$$

where $\left(1-w q^{ \pm 1} / z\right)^{-1}$ denotes the formal infinite series. Similar formulas for $X_{i}^{ \pm}(z) X_{j}^{ \pm}(w)$ imply relation 2.24 . We also use the latter formulas to prove relation 2.25 . The normal ordering yields six products of infinite series which cancel in a remarkable way. We finally arrive at the following.

THEOREM 1. The basic representations of the quantum affine algebra $\mathrm{U}(\hat{\mathrm{g}})_{\mathrm{q}}$ in the spaces $\mathrm{V}_{\mathrm{r}}$ are given by the following operators:

$$
\begin{aligned}
\gamma & \mapsto q(\mathrm{c} \mapsto 1), \quad \mathrm{x}_{\mathrm{in}}^{ \pm} \mapsto \mathrm{X}_{\mathrm{i}}^{ \pm}(\mathrm{n}) \\
\varphi_{\text {in }} & \mapsto \Phi_{\mathrm{i}}(\mathrm{n}), \quad \psi_{\text {in }} \mapsto \Psi_{\mathrm{i}}(\mathrm{n}) .
\end{aligned}
$$

The representation $\mathrm{V}_{\mathrm{r}}$ is irreducible and its highest weight vector with respect to $\mathrm{U}(\hat{\mathrm{g}})_{\mathrm{q}}$ is $1=1_{\mathrm{S}} \otimes 1_{\mathrm{Q}}$ with the same highest weight as in the Lie algebra case.

Thus the above representation of $U(\hat{\mathrm{g}})_{q}$ in $V_{r}$ is in fact a $q$ deformation of the corresponding basic representation of the affine Lie algebra $\hat{\mathrm{g}}$.

We note that when $q$ is a root of unit, expression 3.11 for the vertex operators does not make sense. We can modify $\alpha_{i}(n), i=1, \ldots, l, n \neq 0$, by introducing the operators

$$
\tilde{\alpha}_{i}(n)=\frac{n t}{q^{n}-q^{-n}} \alpha_{i}(n) .
$$

Then the vertex operators are well-defined in terms of the modified Heisenberg algebra even when $q$ is a root of unit. However, the representation $V_{r}$ is no longer irreducible.

Section 4. Quantum Analogue of a Chevalley Basis for $\widehat{\mathfrak{G l}}(n)$

The quantum affine algebra $U(\hat{\mathrm{g}})_{q}$ is defined above as a quantum analogue of the universal enveloping algebra $U(\hat{\mathrm{g}})$ of the affine Lie algebra $\hat{\mathrm{g}}$ in terms of generators $\mathbf{2 . 1}$ and relations 2.2-2.6. One can view $\hat{g}$ with a preferred basis, such as a Chevalley basis, leading to an alternative realization of $U(\hat{\mathrm{g}})$ in terms of generators given by the basis and relations given by the Lie bracket. In general the quantum analogue of $\hat{g}$ is not known. However, Drinfeld's realization is a major step in this direction, and in the case of $\hat{\mathrm{g}}=\widehat{\mathfrak{S l}}(2)$ provides the solution. One of the advantages of a quantum analogue of $\hat{g}$ would be the elimination of the cubic (or higher order) relations such as 2.25 unavoidable in Drinfeld's realization. Using the vertex representation we are able to construct a quantum analogue of a Chevalley basis of $\widehat{\mathfrak{g l}}(n)$ and find the relations which are valid in the basic representations.

We choose a basis $\left\{\alpha_{1}, \ldots, \alpha_{l}\right\}$ of the root system of type $A_{l}$, so that for $i, j=1, \ldots, l, i \neq j$,

$$
\left\langle\alpha_{i}, \alpha_{j}\right\rangle=\left\{\begin{aligned}
-1, & i-j= \pm 1 \\
0, & i-j \neq \pm 1
\end{aligned}\right.
$$

We also define a skew symmetric bilinear form $\{\cdot, \cdot\}: Q \times Q$ $\rightarrow \mathbb{Z}$ as follows:

$$
\left\{\alpha_{i}, \alpha_{j}\right\}=\left\{\begin{aligned}
\pm 1, & i-j= \pm 1 \\
0, & i-j \neq \pm 1
\end{aligned}\right.
$$

For $a=a_{i} a_{i+1} \ldots a_{i+s}, \alpha=\bar{a}=\alpha_{i}+\alpha_{i+1}+\ldots+\alpha_{i+s}, 0$ $\leq s \leq l-i$, we define

$$
\begin{aligned}
X^{+}(a, z) & =: X_{i}^{+}\left(z q^{i}\right) \ldots X_{i+s}^{+}\left(z q^{i+s}\right): \\
X^{-}\left(a^{-1}, z\right) & =: X_{i+s}^{-}\left(z q^{i+s}\right) \ldots X_{i}^{-}\left(z q^{i}\right): \\
\Phi(\alpha, z) & =\Phi_{i}\left(z q^{i}\right) \ldots \Phi_{i+s}\left(z q^{i+s}\right) \\
\Psi(\alpha, z) & =\Psi_{i}\left(z q^{i}\right) \ldots \Psi_{i+s}\left(z q^{i+s}\right) .
\end{aligned}
$$

For $a$ and $\alpha$ as above we also define

$$
\begin{aligned}
X^{-}(a, z) & =\left.X^{+}(a, z)\right|_{t \rightarrow-t} \\
X^{+}\left(a^{-1}, z\right) & =\left.X^{-}\left(a^{-1}, z\right)\right|_{t \rightarrow-t} \\
\Phi(-\alpha, z) & =\left.\Phi(\alpha, z)\right|_{t \rightarrow-t} \\
\Psi(-\alpha, z) & =\left.\Psi(\alpha, z)\right|_{t \rightarrow-t}
\end{aligned}
$$

Finally we set

$$
X^{ \pm}(-a, z)=-X^{ \pm}(a, z) .
$$

Thus we have defined $\Phi(\alpha, z), \Psi(\alpha, z)$ for every $\alpha \in \Delta$ and $X^{ \pm}(a, z)$ for every $a \in Q$ such that $\bar{a} \in \Delta$. We also define their homogenous components $\Phi(\alpha, n), \Psi(\alpha, n)$ and $X^{ \pm}(a$, $n), n \in \mathbb{Z}$, by formulas similar to $3.4,3.5$, and 3.12.

Remark: One notices that we have a doubled set of vertex operators corresponding to the roots in $\Delta$. However, in the limit $q \rightarrow 1(t \rightarrow 0)$ both vertex operators $X^{ \pm}(a, z)$ converge to the same limit, which is the vertex operator in the affine Lie algebra representation.

Let us introduce the notation

$$
\tilde{g}_{\alpha \beta}(z)=g_{\alpha \beta}\left(z q^{\langle\alpha, \beta\rangle\{\alpha \beta\}}\right) .
$$

Then we obtain the following.

THEOREM 2. (i) The operators

$\left\{\mathrm{X}^{ \pm}(\mathrm{a}, \mathrm{k}), \Phi(\alpha, \mathrm{m}), \Psi(\alpha, \mathrm{n}) \mid \alpha, \overline{\mathrm{a}} \in \Delta\right.$;

$$
\left.\mathrm{k} \in \mathbb{Z}, \mathrm{m} \in-\mathbb{Z}_{+}, \mathrm{n} \in \mathbb{Z}_{+}\right\}
$$


in the basic representation of the quantum affine algebra $\mathrm{U}(\widehat{\mathfrak{I}}(1+1))_{\mathrm{q}}$ satisfy the following relations, written in terms of their generating functions, for $\mathrm{a}, \mathrm{b} \in \hat{\mathrm{Q}}, \overline{\mathrm{a}}=\alpha, \overline{\mathrm{b}}=\beta \in \Delta$ :

$$
\begin{aligned}
& \mathrm{X}^{+}\left(\mathrm{a}, \mathrm{zq}^{-1}\right)=\Phi\left(\alpha, \mathrm{zq}^{-1 / 2}\right) \mathrm{X}^{-}(\mathrm{a}, \mathrm{z}) \\
& \mathrm{X}^{+}(\mathrm{a}, \mathrm{zq})=\mathrm{X}^{-}(\mathrm{a}, \mathrm{z}) \Psi\left(\alpha, \mathrm{zq}^{1 / 2}\right) \\
& \Phi(\alpha, \mathrm{z}) \Phi(\beta, \mathrm{z})=\Phi(\alpha+\beta, \mathrm{z}), \alpha+\beta \in \Delta \\
& \Psi(\alpha, \mathrm{z}) \Psi(\beta, \mathrm{z})=\Psi(\alpha+\beta, \mathrm{z}), \alpha+\beta \in \Delta \\
& \Phi(\alpha, \mathrm{z}) \Psi(\beta, \mathrm{w}) \Phi(\alpha, \mathrm{z})^{-1} \Psi(\beta, \mathrm{w})^{-1} \\
& =\tilde{\mathbf{g}}_{\alpha \beta}\left(\mathrm{zw}^{-1} \mathrm{q}^{-1}\right) / \tilde{\mathbf{g}}_{\alpha \beta}\left(\mathrm{zw} w^{-1} \mathrm{q}\right) \\
& =\tilde{\mathrm{g}}_{\alpha \beta}\left(\mathrm{zw}^{-1} \mathrm{q}^{ \pm 1 / 2}\right)^{ \pm 1} \mathrm{X}^{ \pm}(\mathrm{b}, \mathrm{w}) \\
& =\tilde{\mathrm{g}}_{\alpha \beta}\left(\mathrm{z}^{-1} \mathrm{wq}^{\mp 1 / 2}\right)^{\mp 1} \mathrm{X}^{ \pm}(\mathrm{b}, \mathrm{w}) \\
& {\left[\mathrm{X}^{+}(\mathrm{a}, \mathrm{z}), \mathrm{X}^{-}(\mathrm{b}, \mathrm{w})\right]=0,\langle\alpha, \beta\rangle \geq 0} \\
& \int \delta\left(\mathrm{zw}^{-1} \mathrm{q}\right) \Phi\left(\beta, \mathrm{zq}^{1 / 2}\right) \mathrm{X}^{+}(\mathrm{ab}, \mathrm{z}) \quad\langle\alpha, \beta\rangle=\{\alpha, \beta\}=-1 \\
& \delta\left(z w^{-1} q^{-1}\right) X^{+}(a b, z) \Psi\left(-\beta, z^{-1 / 2}\right) \\
& \langle\alpha, \beta\rangle=-\{\alpha, \beta\}=-1 \\
& \left\{\delta\left(\mathrm{zw}^{-1} \mathrm{q}^{-1}\right) \Psi\left(\alpha, \mathrm{wq}^{1 / 2}\right)\right. \\
& \left.-\delta\left(\mathrm{zw}^{-1} \mathrm{q}\right) \Phi\left(\alpha, \mathrm{zq}^{1 / 2}\right)\right\} /\left(\mathrm{q}-\mathrm{q}^{-1}\right)
\end{aligned}
$$

(ii) Let $\mathrm{U}(\widehat{\text { Il }}(1+1))_{\mathrm{q}}^{\text {basic }}$ be an associative algebra with unit 1 , generators 4.13, and relations 4.14-4.23. Then the linear map

$$
\begin{aligned}
\gamma & \mapsto(c \mapsto 1), \quad x_{\text {in }}^{ \pm} \mapsto X^{ \pm}\left(a_{i}^{ \pm 1}, n\right) q^{\text {in }} \\
\varphi_{\text {in }} & \mapsto \Phi\left(\alpha_{i}, n\right) q^{\text {in }}, \quad \psi_{\text {in }} \mapsto \Psi\left(\alpha_{i}, n\right) q^{\text {in }}
\end{aligned}
$$

defines a surjection of $\mathrm{U}(\widehat{\mathfrak{G} l}(1+1))_{\mathrm{q}}$ onto $\mathrm{U}(\widehat{\mathfrak{G} l}(1+1))_{\mathrm{q}}^{\text {basic }}$

The surjection in terms of the original generators 2.1 of $U(\mathfrak{\mathfrak { I } l}(l+1))_{q}$ admits a simple description using the vertex operators corresponding to the maximal root and its nega- tive. As in Drinfeld's realization (10) one has $k_{i} \mapsto \Psi\left(\alpha_{i}, 0\right)$, $k_{0}^{-1}=\Phi\left(\alpha_{i}, 0\right), x_{i}^{ \pm} \mapsto X^{ \pm}\left(a_{i}^{ \pm 1}, 0\right), k_{0} \mapsto q \Phi\left(\alpha_{\max }, 0\right), k_{0}^{-1} \mapsto$ $q^{-1} \Psi\left(\alpha_{\max }, 0\right)$. The explicit expressions for $x_{0}^{ \pm}$are as follows: $x_{0}^{+} \mapsto X^{+}\left(a_{\max }^{-1}, 1\right), x_{0}^{-} \mapsto X^{-}\left(a_{\max },-1\right)$, where $a_{\max } \in$ $\hat{Q}$ is such that $\bar{a}_{\max }=\alpha_{\max }$.

We thank E. Witten for his interest in this work and for discussions of its applications to quantum field theory. I.B.F. gratefully acknowledges support from the Institute for Advanced Study, the Alfred P. Sloan Foundation, and National Science Foundation Grant DMS-8602091.

1. Drinfeld, V. G. (1986) Proc. ICM (Am. Math. Soc., Berkeley, CA), pp. 798-820.

2. Drinfeld, V. G. (1985) Dokl. Akad. Nauk SSSR 283, 1060-1064 [(1985) English transl.: Sov. Math. Dokl. 32, 254-258].

3. Jimbo, M. (1985) Lett. Math. Phys. 10, 63-69.

4. Faddeev, L. D., Reshetikhin, N. Y. \& Takhtajan, L. A. (1987) Quantization of Lie Groups and Lie Algebras, LOMI, Preprints E-14-87.

5. Kac, V. G. (1967) Funkts. Anal. Prilozh. 1, 82-83 [(1967) English transl.: Funct. Anal. Appl. 1, 328-329].

6. Moody, R. V. (1967) Bull. Am. Math. Soc. 73, 217-221.

7. Kantor, I. L. (1968) Dokl. Akad. Nauk SSSR 179, 534-537 [(1968) English transl.: Sov. Math. Dokl. 9, 409-412].

8. Kac, V. G. (1974) Funkts. Anal. Prilozh. 8, $77-78$ [(1974) English transl.: Funct. Anal. Appl. 8, 68-70].

9. Lusztig, G. (1988) Adv. Math. 70, 237-249.

10. Drinfeld, V. G. (1987) Dokl. Akad. Nauk SSSR 296, 13-17 [(1987) English transl.: Sov. Math. Dokl. 36, 212-216].

11. Frenkel, I. B. \& Kac, V. G. (1980) Invent. Math. 62, 23-66.

12. Segal, G. (1981) Commun. Math. Phys. 80, 301-342.

13. Lepowsky, J. \& Wilson, R. L. (1978) Commun. Math. Phys. 62, 43-53.

14. Frenkel, I. B. (1986) Proc. ICM (Am. Math. Soc., Berkeley, CA), pp. 821-839.

15. Borcherds, R. E. (1986) Proc. Natl. Acad. Sci. USA 83, 30683071.

16. Frenkel, I. B., Lepowsky, J. \& Meurman, A. (1988) Vertex Operator Algebras and the Monster (Academic, New York), in press.

17. Witten, E. (1986) Proc. ICM (Am. Math. Soc., Berkeley, CA), pp. 267-303.

18. Friedan, D., Qiu, Z. \& Shenker, S. (1984) Proceedings of the 1983 MSRI Conference, eds. Lepowsky, J., Mandelstam, S. \& Singer, I. M. (MSRI, Springer, New York), pp. 419-449.

19. Goddard, P., Kent, A. \& Olive, D. (1985) Phys. Lett. B 152, 88-92. 